\title{
Correcting an Aberration with a Wire Corrector for SEM
}

\author{
Tomonori Nakano ${ }^{1}$ and $\mathrm{Yu}$ Yamazawa ${ }^{2}$. \\ 1. Research \& Development Group, Hitachi, Ltd., Kokubunji, Japan. \\ 2. Hitachi High-Technologies, Hitachinaka, Japan.
}

Aberration correctors are applied to transmission electron microscopes (TEM) and scanning TEMs (STEM) for high-resolution observation and material analysis [1]. Conventional aberration correctors mostly consist of magnetic poles made of materials with a high magnetic permeability. Those materials are also prone to magnetic hysteresis and inhomogeneity. A number of iterations are therefore required to correct the aberration, even in predetermined conditions. To avoid this issue, a wire corrector was developed [2]. The wire corrector uses symmetric parallel lines (a wire lens) which allow the current to generate multipole fields without using any magnetic materials. Optical simulations have previously suggested the wire corrector can correct spherical aberration [3-4]. In this study, a demonstration of the aberration correction using a prototype wire corrector installed in a conventional scanning electron microscope (SEM) reveals the wire corrector is a practical possibility.

In the wire lens, the magnetic multipole fields including the hexapole field are generated by currents running through symmetric parallel lines alongside the axis, as shown in Fig. 1 (a). The wire lens is shown in Fig. 1 (b). The parallel lines are arranged around the center hole of the wire lens. Although only six lines are needed to generate the hexapole field, 24 lines are provided to increase the intensity and approximate the ideal field-distribution. As shown in Fig. 1 (c), a typical spherical aberration correction setup, in which a pair of hexapoles (Hex1 and Hex2) and transfer doublets (TL1 and TL2) are placed between two condenser lenses (CLs), is applied to a conventional SEM; S-4300 (Hitachi HighTechnologies Corp.) for an aberration correction system.

Figure 2 shows SEM images before and after aberration correction. The specimens are gold-coated latex balls on silicon substrate. These images are obtained under the same conditions (Vacc: $30 \mathrm{kV}$, WD: 6 $\mathrm{mm}$, aperture angle: $9 \mathrm{mrad}$ ). To confirm that the aberration has been corrected, the aperture angle is increased from the optimum aperture angle $(3 \mathrm{mrad})$. Comparison of the two images in Fig. 2 reveals that fine structures on the surface of the latex balls are observable after the aberration correction. This result indicates that the wire corrector can compensate for aberration and improve the SEM resolution.

\section{References:}

[1] H. Rose, Nuclear Instruments and Methods in Physics Research 187 (1981), p. 187.

[2] H. Ito et al, U.S. patent 7,872,240 (2011)

[3] S. Hoque et al, Ultramicroscopy 161 (2016), p. 74.

[4] T. Nakano and Y. Yamazawa, Journal of Vacuum Science \& Technology B 37 (2019), p. 012901. 

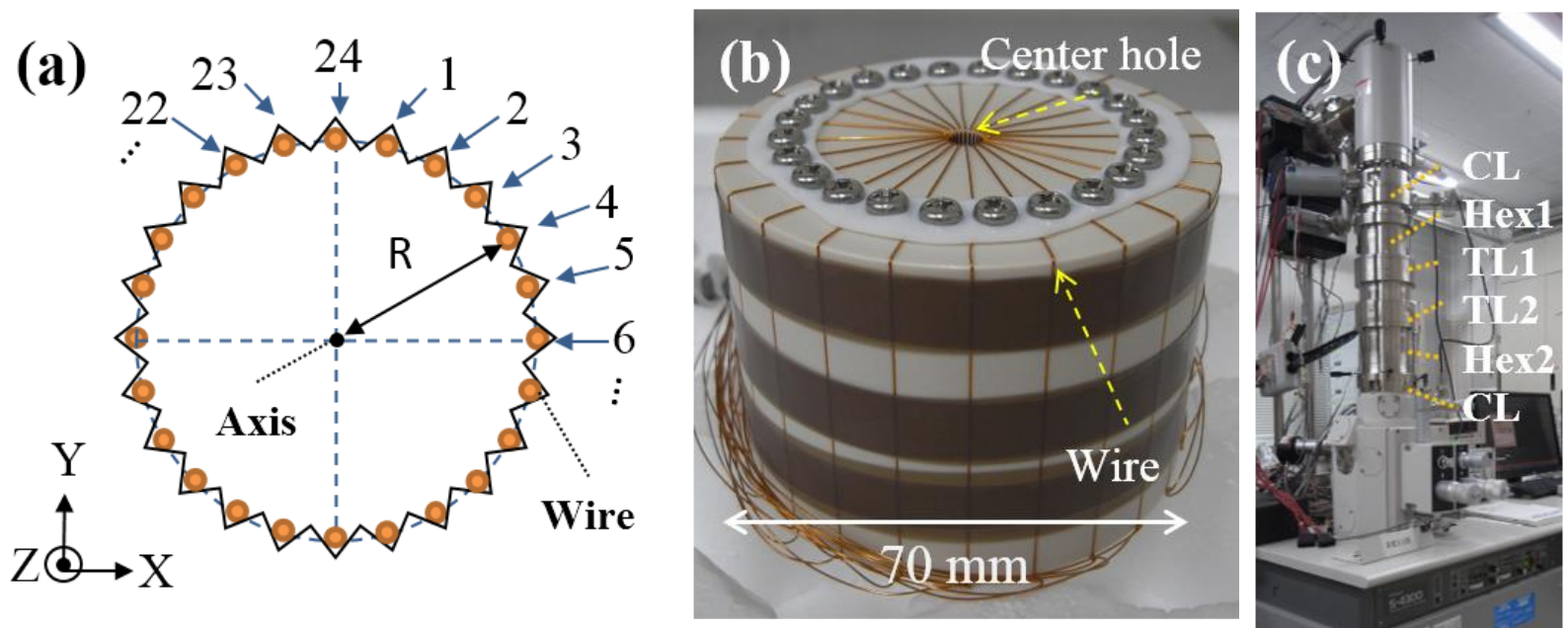

Figure 1. (a) Wire lens schematic (b) Wire lens installed in hex chamber (c) Overall appearance of the experimental apparatus.
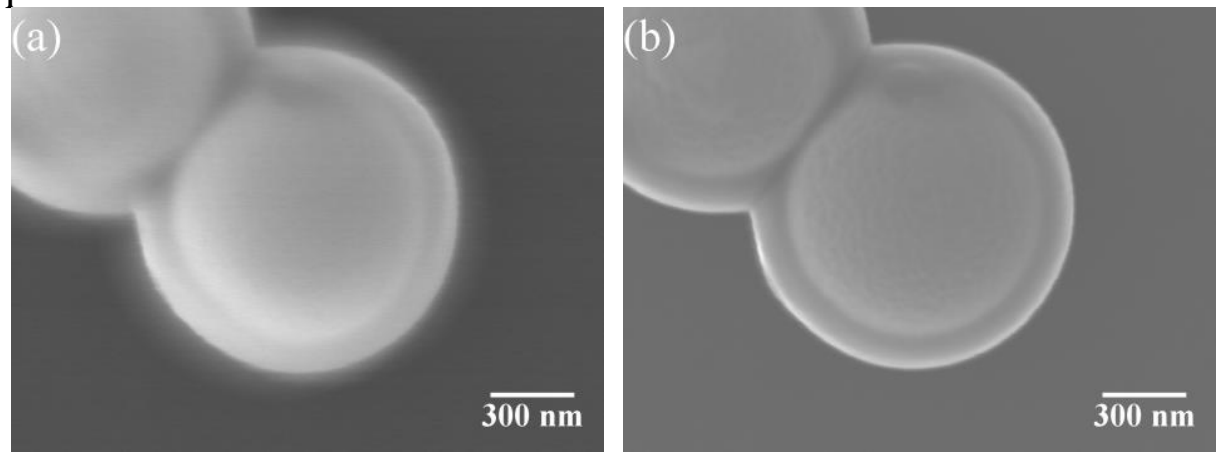

Figure 2. SEM images before (a) and after (b) aberration correction by wire corrector. Acceleration voltage is $30 \mathrm{kV}$, and aperture angle is $9 \mathrm{mrad}$. 